\title{
GBG Approach for Connectivity and Coverage Control in Wireless Sensor Network
}

\author{
Buddha Singh \\ School of Commputer \& \\ System Science, Jawaharlal \\ Nehru University, New Delhi, \\ India.
}

\author{
Haider Raza \\ Dept of Computer Science \& \\ Engg, Manav Rachna \\ International University, \\ Faridabad, India.
}

\author{
Ritu \\ Dept of Computer Science \& \\ Engg, Manav Rachna \\ International University, \\ Faridabad, India.
}

\begin{abstract}
Wireless sensor network are used to monitor various physical environment. These environments are mostly highly asymmetric terrains. The deployment of the sensor node for coverage and connectivity cost should be optimized. Therefore, in sensor network, providing high connectivity and coverage becomes the critical issue. In the proposed work, coverage and connectivity is a minimization of cost function as square of the distance between the nodes. This has been solved for cost optimization which is based on gradient methods. This modified protocol provides a genetic framework. We assume that the backbone nodes are connected to each and they provide coverage to the sensors on the basis of fitness function. The protocol performing better on the basis of cost which is also optimizing both network coverage and backbone connectivity. Our formalization allows the design of self-organized network system, which achieves minimum cost configurations. We have presented simulation results that show the effectiveness of genetic algorithm to provide network configuration that optimize both network coverage and backbone connectivity in different scenarios. We have simulated the Genetic Based Gradient (GBG) method and the proposed work in done on MATLAB.
\end{abstract}

\section{Keywords}

Genetic based gradient (GBG), Genetic Algorithm, Coverage and Connectiviy.

\section{INTRODUCTION}

A Wireless Sensor Network (WSN) is a special kind of a mobile ad hoc network composed of tiny devices with communication and processing capabilities, called sensor nodes.

It consists of three types of nodes: sink nodes, sensor nodes, and relay nodes. A sensor node is a gadget which measures a physical quantity in its surrounding fields and performs the duty of reposting it to a distant observer or an instrument. Relay nodes receive data and forward it to another accessible relay node, according to routing policy of the network with the intention of finally reaching the sink node. The sink node acts as base station in cellular network, It collects all the data from relay nodes and sends it to a home base station where the data is processed [11].

The major problem with wireless sensor networks is limited source of energy, the coverage constraint and lifetime of the wireless sensor network which depend on the power used by the nodes to transmit their data.
The term coverage can have different meanings such as field coverage, target coverage or path coverage shown in Fig. 1. The two fundamental issues are coverage and connectivity, which are considered to be a measure of Quality of Service (QoS) and are related to energy saving, connectivity, network reconfiguration etc. In the field coverage, the issue is to cover all the fields. The target coverage involves covering a set of discrete points in the field and barrier coverage involves covering all the point considered. The Coverage and Connectivity issues affect the cost and performance of wireless sensor networks. Therefore, determining a minimum number of sensors that covers the target region or objects is always a challenging research problem. Further to disseminate the information gathered by a sensor needs connectivity among the nodes. Therefore, ensuring connectivity in a static sensor network is very important task. However, in dynamic sensor network, same problem of connectivity can be realized as a problem of guaranteed delivery. Both these problems require optimal solution in terms of minimum number of sensors and maximum connectivity among the nodes.

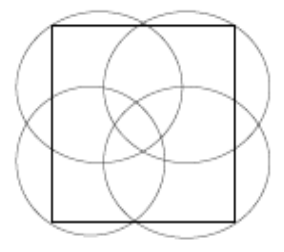

Field coverage

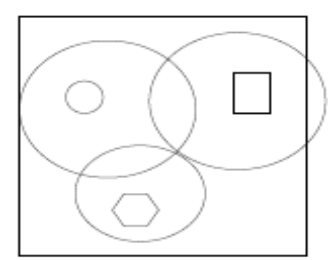

Target coverage

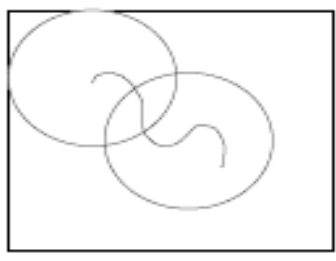

Path coverage

Fig.1 Different types of coverage

\section{RELATED WORK}

A number of solutions are proposed by various researchers related to the coverage and connectivity optimization problems based on location, deployment and tracking of sensor network. The 
problem of how well a given area can be monitored is termed as coverage, the manner in which sensors are placed in the physical environment in order to meet quality requirements is a significant facet of coverage,

- If less sensor nodes are deployed than the required number, blind areas may emerge, leading to inaccurate tracking or monitoring of the area covered by the sensor network.

- If we increase the density of the network by use of redundant sensors then redundant sensor may improve coverage but they will also lead to energy consumption and therefore, reduce network lifetime.

One of the solutions is intersection point method to overcome blind areas in deployment of sensors [6].

Chakarabarty has given an Integer Linear Programming (ILP) Model that minimizes the cost of heterogeneous sensor nodes, and guarantees sensor field coverage [9]. Their problem is defined as the placement of sensor nodes on grid points, and they propose two approaches: a minimum-cost sensor placement, and a sensor placement for target location.

The dynamic multi-product problem of facilitates location is formulated for hinojosa, Puerto, and Fern'andez in a mixed integer linear Programming Model [10]. In this work the objective is to minimize the cost.

The problem of lower bound is obtained by Lagrangian Relaxation. With this solution a heuristic is used to obtain feasible solutions. Similarly, in a flat network architecture, since all wireless nodes are homogenous in terms of their communication capabilities and throughput capacity, flat wireless network architecture without an infrastructure support is not scalable as the number of nodes becomes large so in order to meet the increasing demand of wireless users, it is necessary to supplement the wireless network with a higher level of communication mode. But coverage and connectivity should be minimized with improved throughput so another proposed solution is gradient based approach to solve the joint coverage-connectivity optimization problem that reacts to external forces in order to minimize potential energy [1]. Here the quadratic cost function for both coverage and connectivity is defined in terms of the square distances between neighbour nodes, which are related to the actual energy usage of the network system. The net force on a backbone node is defined as the energy gradient at the location of the backbone node, and only depends on neighbour's position information.

\subsection{Quadratic Minimization Problem}

The problem of how well a given area can be monitored is termed as coverage. For quadratic minimization problem, assume that there is a network of $N$ wireless end hosts and $M$ backbone nodes placed in a geographical area $A$, that is a subset of in the plain. The end hosts are at locations $r_{1}, r_{2} \times m$ min the network in which $=C x_{i x_{s}^{a}}$ represents the location of the end host in $A$. Similarly, the backbone nodes are at locations $R_{1}, R_{2}, R_{9} \ldots$ in the network, in which $=\left(A_{j}\right.$ ? denotes position of the $j$ backbone node in $A$. Each backbone node provides coverage to a collection of end hosts in its proximity. In this process a host $S$ is communicating with another host $D$ in the following way: host $S$ is transmitting its information to the nearby backbone node, and then the traffic traverses through the backbone network until it reaches the backbone node that is closest to the destination. Finally, the backbone node that is closest to the destination transports the traffic to the host $D$.



\subsection{Coverage Cost}

The cost of coverage is defined as a function of the distance from individual end hosts to the backbone nodes. The coverage of the end host is inversely proportional to the distance of the end hosts and the backbone nodes. This implies that:

$$
\hbar_{1}=\sum_{i=1}^{M}\left|\pi_{I}-R_{(h, t)}\right|^{2}
$$

In which $\mathrm{J} 1$ represents the coverage cost. This cost function is associated to the energy used by the backbone network to wrap the terminal nodes, which is also a function of the squared distance between each terminal node and is assigned backbone node [1].

\subsection{Connectivity Cost}

In the simplest form, backbone nodes may be connected to form topology, which implies that a chain of links starts to form a backbone node and passes through all the other backbone nodes. In many useful applications such a simple graph is exposed to link failures, if a single link fails the network becomes disconnected. In practice, it is required to achieve some degree of error tolerance by superimposing a redundant connectivity graph among the backbone nodes. For a given connected topology, the connectivity term of the cost function models the distance between neighbour backbone nodes. The cost function is inversely proportional to the distance of nodes far away from each other. Similar to the coverage cost, the quadratic form for the connectivity cost is defined as:

$$
J_{2}=\sum_{j=1}^{M} \sum_{k=1}^{M} \mid B_{j}-B_{k i}^{2} H_{j k}=\operatorname{trn}(A
$$

In which $j_{2}$ represents the connectivity cost, $\operatorname{tr}($.) denotes the trace of a square matrix, and $\mathrm{H}$ is an $\mathrm{M} * \mathrm{M}$ matrix that represents the backbone connectivity graph, i.e., $\quad l=1$ if there exists a link between backbone nodes $\mathrm{j}$ and $\mathrm{k}$. Which is related to the total energy usage for the backbone network? The optimization in the above problem is on values (e.g., through a controlled movement of the backbone nodes). The first term in the above cost function tries to spread the backbone nodes in the network so that the end hosts are well covered by the backbone network, while the second term in the above optimization problem makes an effort to bring the backbone nodes as close as possible to each other so that they can form a strong connected graph. In the above cost function

is a positive coefficient that balances the comparative importance of the two criteria. For a small the coverage is more essential, while a large gives a higher priority to connectivity and tries to keep the backbone nodes close to each other. The approach to solve the optimization problem is to use iterations on the placement of the backbone nodes. The above cost function is quadratic (and hence convex) in the location of each of the backbone nodes [1].

\subsection{Gradient Approach}

The gradient of the cost function with respect to helps in finding the relocation directions of the backbone node $j$. The gradient of 
cost function $J$ with respect to the location of the backbone node $j$ is:

$$
\begin{aligned}
& \frac{\partial J}{\partial X_{j}}=\sum_{i=1}^{U}\left(X_{j}-X_{i}\right) 1(h(\hat{i})=j)+\sum_{j} \sum_{k=1}^{M}\left(X_{j}-X_{\mathrm{k}}\right) H_{j k}
\end{aligned}
$$

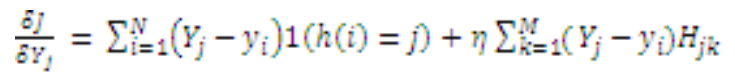

In which 1 (.) is the indicator function, it takes value one if the statement within its argument is true and it is zero otherwise. The above equations can be written in the vector form as follows:

$$
\nabla J_{j}=\sum_{\mathbb{I}}=1\left(R_{j}-R_{i}\right) 1(h(\hat{D})=j)+\sum_{i=1}^{M}\left(R_{j}-R_{R}\right) H_{j k}
$$

In which $T$ is a two dimensional vector that represents the gradient of the cost function $J$ with respect to the location of the backbone node $j$. Since, the gradient is the direction of the steepest ascent of the cost function $J$, relocation of a backbone node in the opposite direction of the gradient gives the steepest drop of the cost function $J$. Therefore, the force at the location of the $j^{\text {th }}$ backbone node can be defined as:

$$
\begin{gathered}
F_{j}=-F j_{j}=\sum_{i=1}^{W}\left(R_{j}-R_{i}\right) 1(h(i)=j)- \\
\sum_{i=1}^{M}\left(R_{j}-R_{\mathbb{N}}\right) H_{j k}
\end{gathered}
$$

On relocating a backbone node in the direction of the force results in the decrease in cost function. An algorithm based on relocating in the direction of the forces stops when the complete value of the force at all backbone nodes is small enough. Also, the force on a given backbone node only depends on the location of its neighbours. Thus, a completely distributed algorithm can be designed where each backbone node react locally to the forces exerted by neighbour nodes, which can provide scalability and robustness. No centralized global information is needed. Each backbone node can make movement decisions by itself informed from purely local information and the overall achieves global optimal configurations. In the next section there is an algorithm for relocating the backbone nodes based on the value of the force at each backbone node. This algorithm also gives a method that assigns each end host to one of the backbone nodes [1].

\subsection{Optimization using Gradient Approach}

Mathematical formulation gives a method for relocating each backbone node in the direction of the force such that cost function shows the steepest descent. It is assumed that for each end host node $i$ the backbone node $h(i)$ is known which provide coverage to the end host node $i$. A set of iterations is used that relocate the backbone nodes [1].

Two operations are used in each relocation iteration: In the first operation the values of the force is used to relocate the backbone nodes and in the second operation, the new locations of the backbone nodes is used to reassign the end hosts to the backbone node, to find the backbone node's new location. Such relocation can be done as follows:

$$
B_{j}^{n+1}=R_{j}^{j}+\delta F_{j}
$$

In which :Denotes the location of the backbone node $j$ at the iteration $n$ and is a small step size, and we have $>0$.

Reassignment of each end host node to the backbone node which has the closest distance with it. For this purpose, we find the following value for each end host node $i^{m} h^{n}(i)=\operatorname{argmin}|l| R_{j}$ - where $1 \leq j \leq M$ in which $h^{n}$ represent the backbone node that provides coverage to the end host node $i$ at iteration $n$, and argmin represent the index $j$ that correspond to the minimum of $\| R_{j}-$ for all $j$. Main focus regarding the above iterations is that in each step of each type of the above iterations, the cost function remains constant or decreases. Note that the first operation decreases the cost function because it relocates a backbone node in the direction of force, which is the opposite direction of the gradient of the cost function.

The second operation further reduces the cost because it reassigns a node $i$ from a backbone node $j$ to another backbone node if and only if $\left\|R_{j}-\gamma_{\mathbb{T}}\right\|>\| R_{j}-\mathrm{a}$, and such an operation either reduces the cost function or does not change its current value. Therefore, this sequence has to coverage to a value as $n$ increases. Following criterion to stop the iteration: $j^{[n+1]}-j<$ in which is a small positive constant. In this, the problem of dynamically optimizing network coverage and backbone connectivity in DWB-based wireless networks is being done by adjusting the location of the backbone nodes.

The gradient based approach in [1] provides a solution to optimize the cost function, that may not convergence fast. Further, the model does not take into account the physical layer issues (i.e. bit error rate and energy consumption) that contribute to the cost of coverage and connectivity.

Therefore, it is important to investigate into these issues to measure the performance of quadratic optimization based model. The joint coverage-connectivity optimization problem is expressed as a quadratic minimization problem which is mentioned in the next section [1].

We have applied Genetic algorithm (GA) for optimization that minimizes the cost of coverage and connectivity. Genetic algorithm is also useful in all the cases irrespective of whether the function is continuous or not therefore, we set the following objectives to carry out the proposed work.

- Implementing the optimization problem using other technique

- Design physical link cost based model

- Simulate physical link cost based model for its performance evaluation in term of energy consumption and bit error.

\subsubsection{Selection}

This operator selects chromosome from population for reproduction, they filter the chromosome the more times it is likely to be select to reproduce.

There are few methods for selection like: Rank Selection, Tournament Selection, Steady State Selection, etc each is having its own merits and demerits, we can choose any of them according to our optimization needs.

\subsubsection{Genetic Operators}

To implement genetic algorithm, we need genetic operators, however the decision depends greatly on encoding strategy. 
Crossover and mutation are discussed here mostly in context of bit string, the whole steps are in Fig. 2

1) Crossover: These operators randomly choose the crossover point and exchange the subsequences before and after that crossover point between two chromosomes to create two offspring. For example the string 10000100 and 11111111 could be crossover after the third bit from left to produce the two offspring 10011111 and 11100100 . The crossover operates roughly by recombination between the two single chromosomes and produces two new offspring in order that they are more fit than their parents.

2) Mutation: This operator randomly flips some of the bit in chromosome, for example, the string 10011111 might be mutated in its fifth position to yield 10010111. Mutation can occur at each bit position in a string a string with some probability, usually very small. Mutation: the mutation is natural process which is responsible new traits appearing within population by random alteration of an individual's code. The mutation can make an individual fitter or not, if it is fit enough, it will pass to the next generation and he is likely to produce otherwise he would disappear from the population at the next generation. The aim of mutation in genetic algorithm is to explore the domain of solutions.

\subsection{ALGORITHM}

We have generated random number of bit length fourteen in which the first seven bits represent $x$ coordinates and the remaining seven bits represent as y coordinates to denote a chromosome.

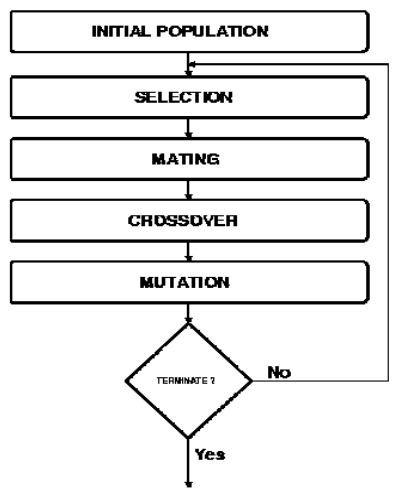

Fig.2 Steps for Genetic Algorithm

This is used both backbone and sensor nodes deployed in two tier structure.

1) We have implemented two functions Chorosomelower and Chormosomeupper generates the random number for representing population in lower and upper tier respectively.

2) Further, we have chosen grid range in such a way that the position of chromosome do not lie outside the grid range.

3) In our problem selection rate is determined based on mutation and crossover rates.

4) The different fitness function parameter are height between the two layer, to provide robustness against failure, we can increase or decrease according to our need.
5) If we need high connectivity as in the case of bad weather, we can take value more than one. Function implementing crossover technique between upper and lower chromosome for the given population with a fixed crossover rate, now we have generated a new population.

6) In this crossover process we are generating two random numbers for a chromosome and exchanging their position with other chromosome., now for mutation the population size chromosomes next lower and next upper,

7) We are doing mutation by inversion of bit at a random position and generating new population. Based on fitness criteria, we are sorting out those pairs for which the connectivity and coverage cost is minimum.

8) We have mapped the lower layer chromosome with upper layer so that each time we get a pair for the fitness criteria. For the value, we have different coverage and connectivity graph and based on this we have calculated cost for various parameters which is shown in simulation and results.

9) The cost function is sum of the square of the distance between the nodes. We have to minimize the cost function using genetic algorithm so that coverage and connectivity remains optimized. We will preserve and add to the population, those chromosomes which follow the fitness criteria.

\section{SIMULATION AND RESULTS}

We have implemented quadratic optimization problem using genetic algorithm on MALTAB and on the basis of proposed work, we may yield better result in terms of convergence

For simulation, we have taken a grid range $=-1$, and generated binary chromosome of length 14 in which the first seven bits denotes $\mathrm{x}$ coordinated and the remaining seven bits represent $\mathrm{y}$ coordinates. As we have taken two-tier structure in which various data for initial population (number of chromosome) and the number of nodes is lower plane and upper plane for two different experiments is as follows:

initPop $=12$, no of chromosomes at the start for lower and upper plane for both experiment.

numModesLower $=16$ and 32 for two experiments, respectively.

numNodesUpper $=8$ and 16 for two experiments, respectively.

mutationRate $=0.25$ and 0.3 for two experiment respectively.

crossoverRate $=0.2$ same for both experiments.

selectionRate $=1 . /(1+$ mutationRate + crossoverRate $)$, same

for both experimernts.

Other fitness function parameters are as follows:

height $=10$ same for both experiments.

et $a=5$ and 1 for two experiments, respectively.

The results of experiments based on above variable and parameters are presented in the following figures.

Figure 3 shows the result for a network with 16 terminal nodes and 8 backbone nodes. The value used for the variable height $=10$ and eta equal to 1 . It shows the network coverage at the expense of low backbone connectivity. The backbone nodes are shown in the figure with square mark (upper layer) and the base nodes (lower layer) with circle and the link between them are shown in the thin lines. Links from each terminal node to its closest backbone node are shown in thinner lines. We assume that the backbone nodes are always connected among themselves. For this deployment the connectivity cost is 129119 square meters, coverage cost is 36627 square meter, the total cost is 165746 square meters. 
Fig. 4 shows the resulting network configuration after running the genetic algorithm for eta equal to 5 for 16 backbone nodes and 8 base nodes, for this deployment the connectivity cost is 27687 square meters, the coverage cost is775059 square meters, total cost is 1209148 square meters. It shows that stronger backbone networks can be used at the experience of lower network coverage.

Fig 5 shows a network with 32 backbone nodes and 16 base nodes. As seen from the figure, the increased number of backbone nodes allows more flexibility to optimize network coverage with less penalization on backbone connectivity and vice versa. The computed connectivity cost is 1271799 square meters, coverage cost is 51045 square meter, the total cost is 1322844 square meters.

Fig 6 shows a network with 32 backbone nodes and 16 base nodes and value of eta is 5 , the calculated connectivity cost is 1322263 square meters, the coverage cost is 123620 square meters, and total cost is 6734930 square meters. It shows that stronger backbone networks can be used at the expense of lower network coverage.

\section{OPTIMAL BACKBONE COVERAGE FOR ETA=1}

\section{- $B A C$ CBONE NODES D TERMNAL NODES}

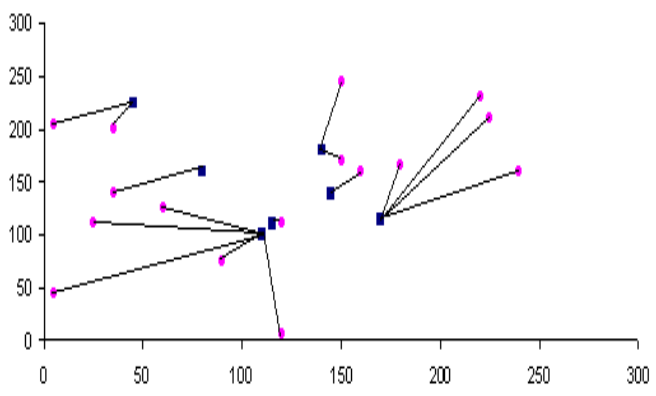

Fig. 3

OPTIMAL BACKBONE COVERAGE FOR ETA=5

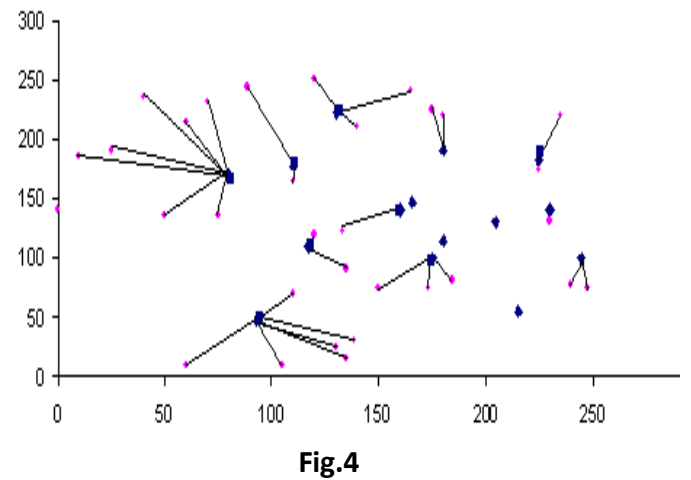

OPTIMAL COVERAGE FOR ETA=5

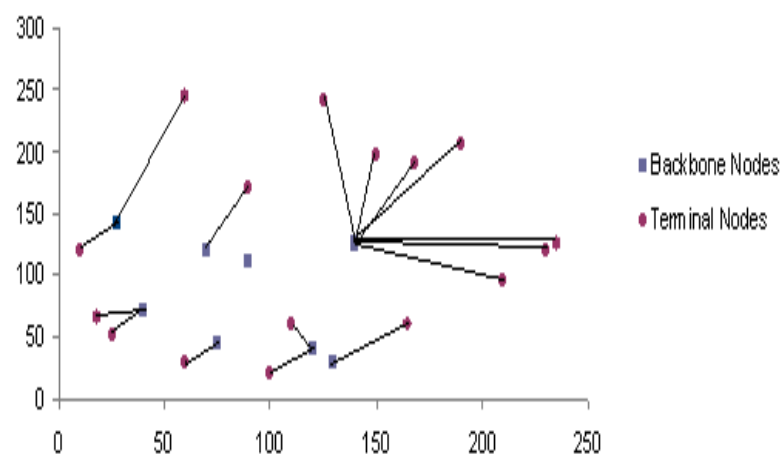

Fig.5

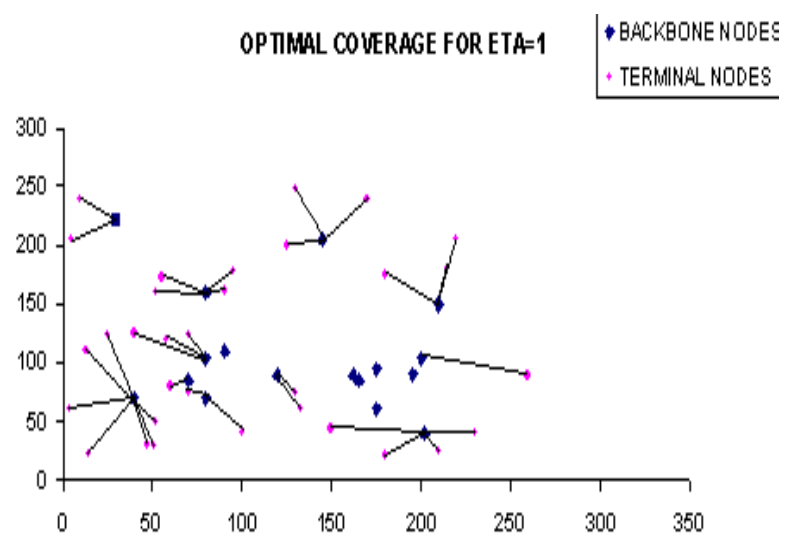

Fig. 6

\section{CONCLUSION AND FUTURE WORK}

We have solved the problem of dynamically optimizing network coverage and backbone connectivity in DWB-based wireless networks using genetic algorithm. This joint coverage coverage-connectivity optimization problem is a quadratic minimization problem which is a quadratic cost function for both coverage and connectivity in term of the square distance between neighbouring nodes. We developed a completely distributed mobility control algorithm based on genetic algorithm that computes the relocation direction of nodes on the basis of current control location. The simulation produces the encouraging results for the cost based control algorithm that optimizes both network coverage and backbone connectivity.

We proposed to use physical link cost models in coverage and connectivity optimization and perform quantitative analysis in terms of energy usage and bit-error rate. Further we would apply other methods of solution for optimization problem that minimize 
the cost of coverage and connectivity and improve energy consumption, throughput and end to end delay.

\section{REFERENCES}

[1] Jaime Liorca, Mehdi kalantari, Stuart D Milner, Christopher C Davis, "A quadratic optimization method for coverage and connectivity control in backbone based wireless sensor networks" IEEE-ISSNIP July 2007, pp 04-25

[2] P.Gupta, P. R. Kumar, "The capacity of wireless sensor networks" ,IEEE Trans.Inform. Theory, Vol. 46, No.2,2000,pp.388-404.

[3] Davis, C., Z. Haas, and S. Milner, "On How To Circumvent The Manet Scalability Curse" In: Proc. IEEE MILCOM, October 2006, pp. 1-7.

[4] T.S. Rapport, wireless communications: principles and practice, prentice hall, Englewood cliffs, $\mathrm{Nj} .1996$.

[5] Llorca, J., A. Desai. And S. Milner, "Obscuration Minimization in Dynamic Free Space Optical Networks through Topology Control", In: Proc. IEEE MILCOM, 2004, Vol. 3, pp. 1247-1253.

[6] Azzedine Boukerche, and Xin Fei "A coverage-preserving scheme for wireless sensor network with irregular sensing range" Elsevier, Ad Hoc Networks(2007) 1303-1316
[7] Atumbe Jules Baruani. Network Engineering Using Multi-Objective Evolutionary Algorithms. Msc, Universit of Stellenbosch, 2007.

[8] WGBH Educational Foundation and Clear Blue Sky Productions. Summary of Darwin's Theory of Evolution.

[9] Chakrabarty, K., Iyengar, S.S., Qi, H., Cho, E.: Grid Coverage for surveillance and target location in distributed sensork networks. In: IEEE Transactions on Computers.(2002) 51(12):1448-1453

[10] Hinojosa, Y., Puerto, J., Fern'andez, F.R.: A multiperiod two-echelon multicommodity capacitaded plant location problem. European Journal of Operational Research 123(2000) 271-291

[11] Jean_Michel Safari Serufuri (jeanmichel@aims.ac.za), Optimisation of Wireless Sensor Network-2008 African Institute of Mathematical Sciences(AIMS).

[12] Genetic algorithms in search,optimization, and machine learningDavid. Goldberg, Addison Wesley. 